\title{
THE NUCLEAR RESEARCH CENTRE, GRENOBLE
}

T

HE Nuclear Research Centre at Grenoble, a further establishment under the control of the French Atomic Energy Commission, was officially opened on January 26.

The creation of this Centre under the French Government's general policy of decentralization is a further step towards increasing the research facilities of the French Atomic Energy Commission.

The Grenoble Centre (C.E.N.G.) will operate in close liaison with the city's University, which possesses laboratories in several branches of science of considerable repute (electrostatics and metal physics, science of low temperatures, fluid mechanics, chemistry, electronics, and so forth) plus excellent engineering faculties, and in particular a special atomic engineering course.

The director of the Centre, Prof. Louis Néel, nember of the Paris Academy of Sciences, is also director of the Ecole Nationale Supérieur d'Ingénieurs of Grenoble and the Electrostatics and Metal Physics Laboratory of the Centre National de la Recherche Scientifique.

The first construction programme for the Nuclear Research Centre at Grenoble was completed in two years, on an extensive site close to the city. The programme comprised a number of modern laboratories suitable for a wide variety of research work, two large hangars of industrial type for noisy or bulky experiments, workshops and general services buildings. Facilities comprise the reactor Mélusine and a group of five electrostatic accelerators. Mélusine owes its name to a water sprite, which according to local tradition haunted the caves of Sassenage, near Grenoble. It is a 20 per cent enriched uranium pool reactor, of $1,200 \mathrm{~kW}$. power, neutron flux $10^{13}$ neutrons $/ \mathrm{cm}^{2} / \mathrm{sec}$. It was built by the French industrial group "Indatom", and the fuel elements are obtained from the United States.

The accelerator building will be equipped in April with one fast neutron generator and four electrostatic accelerators (two ion accelerators, two electron accelerators) with potentials ranging between $600 \mathrm{kV}$. and $1.4 \mathrm{MV}$. Their special characteristic is a very high particle current $(4 \mathrm{~m} . \mathrm{amp}$. with the $1.4 \mathrm{MV}$. ion accelerator) obtained with a type of electrostatic generator designed in one of the laboratories of the University of Grenoble, and built by the S.A.M.E.S., a company specializing in electrostatic machines.

The research programme of the Grenoble Centre is distributed over a number of 'laboratories'. Research in physics includes the activities of the solid state physics laboratory, where the effects of neutron bombardment on matter are studied, in close conjunction with the low-temperature laboratory, ex-

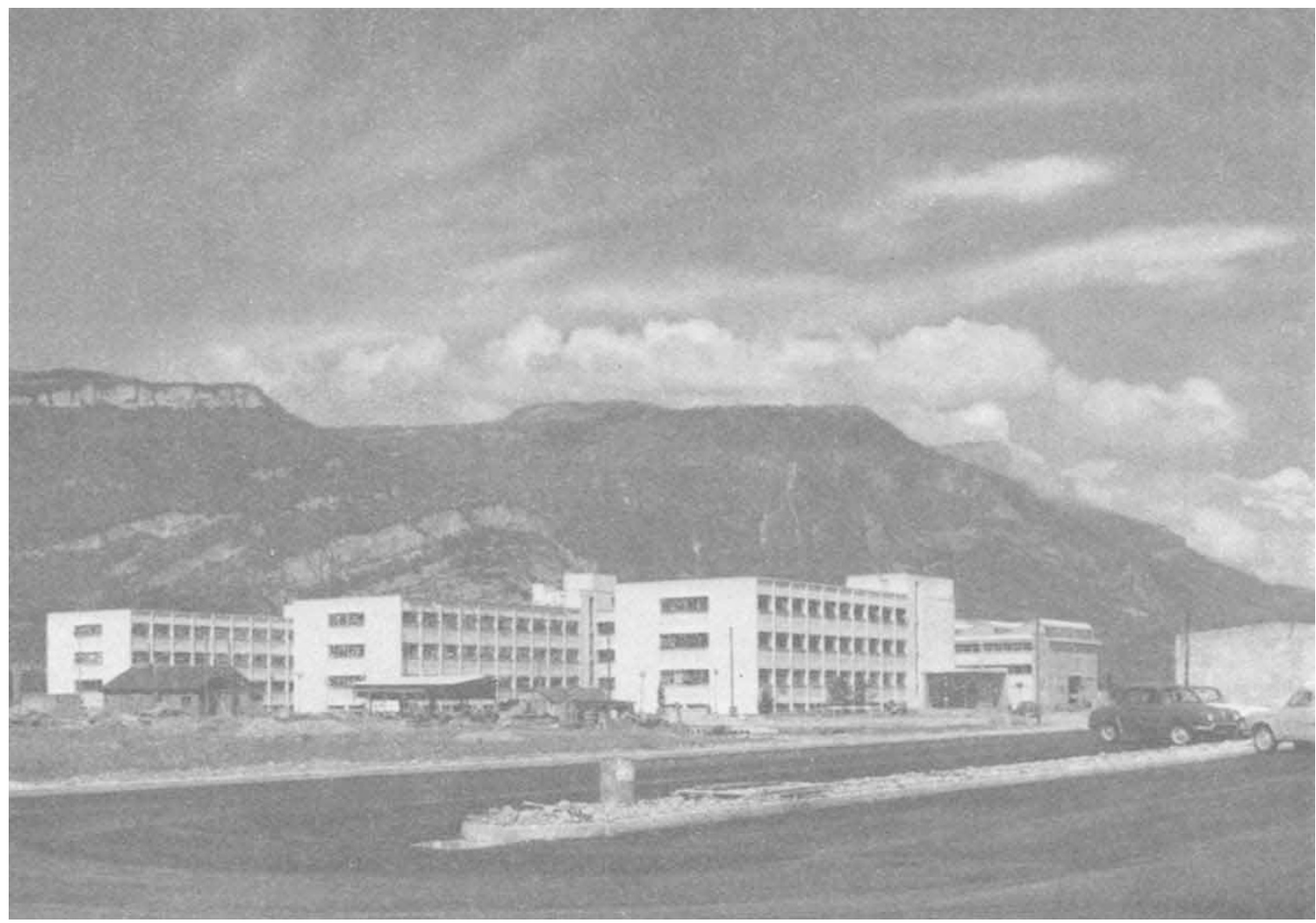

Fig. 1. Laboratory buildings at the Centre d'Etudes Nucléaires de Grenoble 


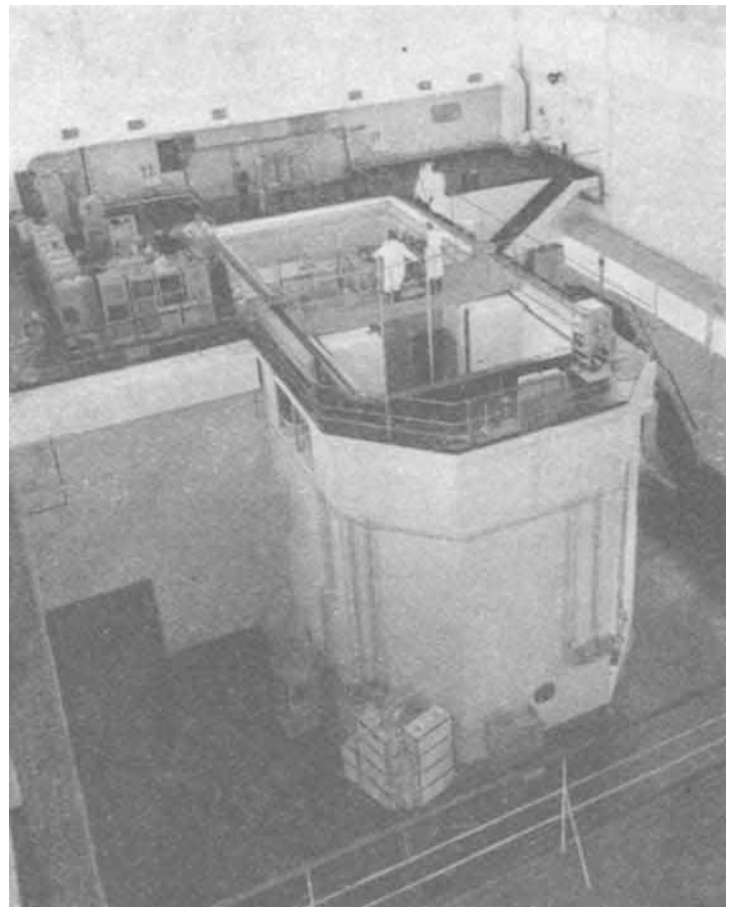

Fig. 2. Swimming pool reactor Mélusine tending this research into the range of $20-900^{\circ} \mathrm{K}$., and also the work of the neutron diffraction and magnetic resonance laboratories.

A radiochemistry laboratory has been installed in the Centre by the French Petroleum Institute, while the chemistry of solids laboratory is working on the production of a uranium compound for nuclear fuel elements.

There are, of course, also electronic laboratories, others for the study of applications of radioelements, and biology and nuclear physics laboratories, and also the thermal exchange laboratory, where heat exchanges with liquids in a reactor are studied.

The Centre's research programme is prepared by a scientific committee composed of six representatives from the Atomic Energy Commission and six professors from the University of Grenoble, who are scientific advisers to the Centre. This ensures the essential co-ordination with the programmes of other Atomic Energy Commission research centres.

As from January 1, the research work of the Centre is employing 300 persons. Construction is continuing, and the total staff by the end of 1961 will be 800 .

In order to help in obtaining the required number of staff, the Centre collaborates with the organizations entrusted with the training of technicians at all levels. It is also pleasant to note that the Centre is situated in a region that is both industrial and of tourist interest, in the heart of the French Alps, and therefore considered particularly attractive by young technicians.

\section{OBITUARIES}

\section{Prof. A. H. Gibson}

Prof. Arnold Hartley Gibson, emeritus professor of engineering in the University of Manchester, died on February 16 at the age of eighty.

Those who have read Prof. Gibson's monograph on Osborne Reynolds will realize how much 'A. H. G.' admired his own teacher. That he shared some of Reynolds's versatility is clear from the fact that although he will best be remembered for his contributions to hydraulics, he also did significant work in structures (the 'circular-arc bow girder') and in applied thermodynamics (many papers on internal combustion engines and heat transmission). At one period he was simultaneously a member of the Air Ministry Engine Research Committee and of the Severn Barrage Committee.

In hydraulics, his investigations ranged from the behaviour of bodies floating in vortices to river models; from water hammer in pipes to the effect of bridge piers on tides ; from centrifugal pumps to the interaction between passing vessels. His famous treatise "Hydraulics and its Applications" was published in 1908 ; the fifth edition appeared in 1952 : he was then seventy-four years old and told me how much he would have liked to rewrite it, but he feared that in that case the price would have been beyond the reach of many for whom the book was intended. The chapter on scale models which he added is probably his last published work.

His writings have an unfailing clarity and directness, virtues which were also very evident in the unaffected elegance of his lectures. He knew just when to stop before confusing the issue.
Gibson's reports on the Severn tidal model (1933) have rightly been described as 'monumental' (an adjective which caused him some amusement). The scope and variety of the experiments-the trials on bed materials, the introduction of silt and the representation of the coagulating effect of the salts of the sea, together with the study of the tides and the bore-remain unsurpassed in this field, even allowing for the later growth in the size of models. His investigations of the Severn and other estuaries revived British interest in the possibilities of scale models as an aid to the design of river and harbour works and led to the creation by the Department of Scientific and Industrial Research of the Hydraulies Research Station at Wallingford.

Generations of students have held him in affection as well as admiration, and one reason for this is to be found in his own interpretation of the duties of a professor. His aim was to inspire and never to 'spoon-feed'. He not only believed that he ought to lecture to each year but also he enjoyed doing so. The course of twenty or twenty-five lectures which he delivered to students in their first term at Manchester, covering a wide field of applied mechanies, brought immediate contact, and this was renewed in the second and third years. At one time or another he lectured on almost every subject in civil and mechanical engineering. Yet in addition he took his full share in the general work of the University in Senate, faculty and committees and maintained his output of important original papers.

The fact that he was able to do so much can be partly explained by his gift for making quick decisions. As Prof. J. A. L. Matheson has written 